\title{
Length-Selective Separation of Cellulose Fibres by Hydrodynamic Fractionation
}

\author{
Published in: Chemical Engineering Research and Design 126, 2017, 54-66
}

http://dx.doi.org/10.1016/j.cherd.2017.08.001

Electronical annex can be found/looked-up at the journal online piblication

Jakob D. Redlinger-Pohn, Josef König, and Stefan Radl

A hydrodynamic fraction device (HDF) was developed based on the principle of spontaneous fibre network formation and subsequent segregation of fibres and fines in cellulose pulp. Separation is most successful in the so-called "annulus plug flow" regime, which is demonstrated for various combinations of fibre concentration and Reynolds number. In this regime, fibres form a network in the channel center, surrounded by fluid with relative low concentration of fibres and large concentration of fines. As in flow channel separation, wall bounded fluid - containing the fines fraction - is removed from the main flow via sidechannels. Long fibres that form a network exit via the main channel. Via an array of experiments we demonstrate precise fractionation of cellulose pulp at a typical cut size of $1 \mathrm{~mm}$. Also, we show that higher Reynolds numbers lead to a dispersion of the fibre network, and consequently a lower sharpness of cut - increasing the fibre concentration leads to a lower yield of shorter fibres. While variation of geometrical parameters did not affect the separation performance, the design of the HDF clearly impacts its capacity. 


\section{Introduction}

Novel and advanced products, and changing economic boundary conditions necessitate rethinking of production processes. In the present study we focus on precise length-based fibre separation, which is a key process in pulp and paper production, as well as future biorefineries for a more complete usage of, e.g., wood [1]. Traditional length-based separation processes include (i) pressure screens, (ii) froth flotation, and (iii) hydro cyclones [2]. Unfortunately, hydro cyclones fractionate according to specific fibre surface and/or fibre coarseness (e.g. vessel cells from hardwood pulp), and flotation add impurities to achieve and maintain a stable froth [3]. Pressure screening sorts fibres according to their probability to follow the fluid flow through a perforated (with holes), or slotted screen. The process was originally developed to remove impurities, i.e. shives, or plastic from pulp suspensions. Flexible and smaller cellulose fibres where accepted through the screen, whilst impurities were rejected [46]. To achieve high capacity, a cylindrical screen is often used, and the pulp suspension is fed from the inside. A rotor with foil elements ensures tangential motion over the screen, and thus cleans the screens from accumulated fibres. Decreasing the hole or slot size leads to lower probability of longer and stiffer fibres being passed. This allows a length-based separation of fibres. Fibre length fractionation using pressure screens has attracted recent research [7-9], with the key conclusions that optimal fibre length fractionation is achieved for (i) small hole and/or slot size, (ii) high reject ratio, and (iii) low fluid velocity in the slot/hole. Unfortunately, those parameters lead to a low separation efficiency (i.e., total removal of solids) and consequently to high suspension thickening. Thus, pressure screens need to be operated in series, and the pulp needs to be re-diluted with water between the operational stages. Also, the rotor of a pressure screen dissipates a significant amount of energy. These drawbacks of pressure screens suggest deeper studies on a more optimal design that (i) dissipates less energy, as well as (ii) allows more controlled and more precise separation. It is exactly this need for research that motivates our present study.

In previous studies, experimentally and numerically, pressure screens have been approximated as a single channel with slots (or holes) on one side of the wall [5,10-13]. While fluid flow profiles have been studied in detail, fibre trajectories have only been studied for dilute suspension with basically no fibre-fibre interactions. Similar geometries were recently studied for their use in micro fluidics [14-17]. Small particles were separated from 
larger and/or longer particles in T-Junction geometries for laminar flow conditions and comparably low particle concentrations. Findings showed, for example, improved lengthbased separation for a backward facing separation channel.

Walmsley and Atkins [18] compared fractionation performance of pressure screens to their approximate: the flow channel. Studies were performed with suspensions involving a (massbased) fibre suspension concentration of $C$ only $0.1 \%$. Their results showed a reduced removal of longer fibres for the flow channel - a more precise length-based separation is thus achieved in simple flow channels. This effect was attributed to different flow patterns. Not mentioned in their study were interactions of fibres, i.e., flocculation, which can already occur at a fibre suspension concentration $C$ of $0.1 \%$. In general, most of the studies on flow channel separation for pressure screening ignored fibre-fibre interactions, focusing only on the motion of individual stiff fibres. In channel flow of a more concentrated suspension, fibres move as (i) plug, (ii) plug with water annulus at the wall, or (iii) fully fluidized in the liquid. The regimes change from (i) to (iii) with increasing Reynolds number $R e$, and/or decreasing concentration $C[19,20]$. Especially longer fibres form flocks, and thus will not move freely across the separation channel. We will show that exactly this flocking behaviour is key for the separation effect in pulp suspensions of comparably high concentration.

In our present contribution we will combine theory on the fractionation of fibres in separation channels with theory on fibre suspension flow in channels. Based on the literature we will present a new separator for precise length-based fibre separation: the hydrodynamic fractionation device (HDF). This paper also includes results from first experimental studies using the HDF: a focus thereby is on the interplay between operation conditions (Reynolds number $R e$ ), the resulting fibre suspension flow regime, and the length-based separation performance. Specifically, we report the separation efficiency, quantified by the grade efficiency, in dependence on:

(i) fibre suspension concentration $C$,

(ii) Reynolds number $R e$ in the main channel,

(iii) relative accept flow rate $\Phi^{+}$, and

(iv) geometrical proportions of the channel: shape of the separation channel and dimension of the main channel cross section. 
Manuscript - Length-Selective Separation of Cellulose Fibres by HDF

\subsection{Outline}

The outline of the paper is as follows:

In section 2 we briefly review literature on flow channels separation and fibre suspension channel flow. We highlight on important aspects and take recommendations for the design of the HDF.

In section 3 we introduce the hydrodynamic fractionation device HDF and detail on its key characteristics. Methods for the investigation and the experimental plan are presented.

In section 4 we report the results from the experimental investigation, and comment on the influence of the tested parameters.

In Section 5 we conclude the present work and propose future work. 


\section{Theory on Fractionation in Channel Flow}

In separation channels, part of the fluid is removed from the main flow via a side channel, which typically spans the full width of the main channel. A simple example is the flow past a T-Junction (see Figure 1). The fluid removed via the side-channel is termed accept, the fluid remaining in the main channel is termed reject. The theory for the separation of fluid and particle in these systems is well established $[4,5,10,11,13,14,16,17,21,22]$, see Figure 1a. The basic assumption is, that the accept flow origins upstream from a layer in the vicinity of the wall. The layer dividing the accept flow and the reject flow is termed exit layer. The height of the exit layer has a complex shape in a three-dimensional channel [23]. However, to a first approximation one can treat the exit layer as parallel to the walls that contain the side channel.

(a)

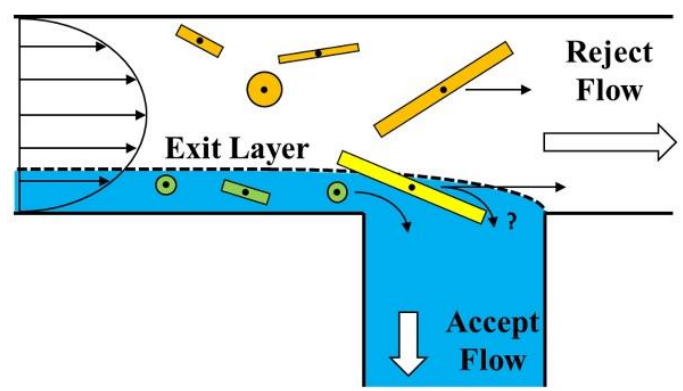

(b)

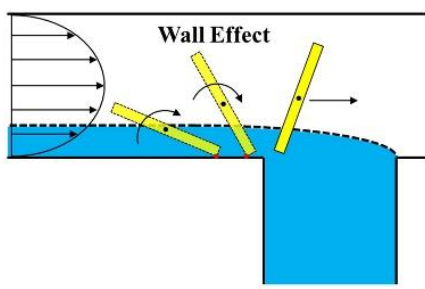

(c)

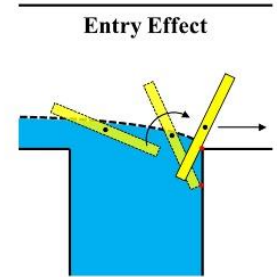

Figure 1: Principle of hydrodynamic fractionation in a separation channel. Accept flow (in blue) and reject flow (in white) are separated by the so-called "exit layer". (a): Particles (green) below the exit layer are removed with the accept flow. Elongated fibres (yellow) are subject to the entry effect discussed in chapter 2.1. Particles and fibres (orange) above the exit layer remain in the reject flow. (b): Turning effect with fibre flipping above the exit layer. (c) Illustration of the entry effect in which a fibre collides with the downstream edge and remains in the reject flow.

The height of the exit layer upstream the separation channel is directly proportional to the accept flow rate. Thus, it can be computed from product of the average side channel velocity and the side channel cross section. Neutrally buoyant particles, that are located below the exit layer upstream of the side channel, will be removed with the accept flow. Particles above the exit layer remain in the reject flow. It follows here as an obvious conclusion, that the exit layer is thicker for higher side channel velocity. Thus, in such a situation more particles are removed, a fact reported for single fibre separation $[4,12,13,24]$. Also, it is well known that the capacity of removing particles from the main flow is indirectly related to the selectivity of 
the separation process. Previous studies on dilute fibre suspension flow gave insight in the separation probability and fluid flow in the separation channel. As a result, different shaped screens, e.g., separation channels with step or slope contours, have been developed $[10,11]$.

\subsection{Dilute Suspensions}

Unlike spherical particles, the probability of separation of elongated particles, e.g., cellulose fibres, also depends on their orientation, their aspect ratio, and the ratio of fibre length to the width of the opening. Early studies [4,18] with stiff fibres in dilute suspension flow, and in which fibre-fibre interactions were absent, identified three responsible mechanisms: (i) the wall effect, (ii) the entry effect, and the so-called (iii) "mat theory".

The wall effect describes the length-dependent concentration gradient of fibres in the vicinity of the wall. Fibres in shear flow perform time dependent rotation [25]. Interactions with the wall during rotation push the fibre away from the wall (illustrated in Figure 1b). The resulting wall normal position depends on the orientation and length of the fibre. For the case of fibres orientated in flow direction, this would be the fibre half-length $[23,26]$. For arbitrary orientated fibres a linear increase in concentration is noted until reaching the fibre half-length $[5,11]$. This fibre length dependent concentration results in fewer longer fibres within the exit layer, benefiting a preferred separation of smaller fibres.

The entry effect applies for stiff fibres within the exit layer that are preferably orientated in flow direction. For a screen opening smaller than the fibre length, the fibre needs to rotate into the separation channel to be accepted. Upon rotation, the fibre can interact with the screen possibly causing the fibre to rotate past the screen (illustrated in Figure 1c). Thus the entry effect describes the inability of the fibre to turn into the accept flow in time. Consequently the fibre will remain in the reject flow. Again, the effect promotes preferential separation of smaller fibres from larger fibres. The effect is less pronounced for flexible fibres, e.g., cellulose pulp fibres, which may bend when entering the separation channel.

The wall and entry effect are also exploited for the separation of different shaped cells [17]. Smaller spherical cells have been successfully separated from elongated bacterial cells which remained in the main channel. 
The so-called "mat theory" considers the stapling of stiff fibres at the screen, and the interference of the fibre mat with fibres in the suspension. Stapling of fibres might occur especially on the down-stream edge of the screen [13]. The fibre mat can reduce the effective opening of the screen, and thus reduces the accept flow and the exit layer thickness. Mat formation on the up- and/or down-stream edge of the separation channel depends on the shape and geometry of the channel (as discussed at the end of this section), accept flow rate, the fibre concentration, and fibre flexibility. Kumar [13], performed experiments with stiff nylon fibres at high and low fibre concentration, and with flexible rayon fibres. The results for the stiff nylon fibres showed a drop in the separation of longer fibre $(3 \mathrm{~mm})$, and a decrease for shorter fibre $(1 \mathrm{~mm})$ at increased concentration. Staples of fibres formed, but were observed to be unstable, i.e., they were washed off from time to time. In contrast, flexible rayon fibres already formed larger flocs up-stream and tended to fully block the separation channel. The fibre mat was stable and resided on the opening of the separation channel fully blocking it. Olson [5] and Dong [11] performed numerical simulations, highlighting that fibres interact with the screen depending on their initial height and position.

\subsection{Dense Suspensions}

Fibres already cluster to flocs and/or form networks at a relatively low mass concentration, which can be well below $1 \%$. Floc formation, and floc stability is found to depend on multiple parameters, including (i) fibre aspect ratio, (ii) fibre flexibility, and (iii) fibre surface roughness including fibrillation [27-32]. Fibres entangle and remain in contact if they are linked at several contact points (where number of contact is often given as 3 [33]) withstanding significant fluid shear. For increasing fluid shear, exceeding the flocs yield stress, fibre disperses again after being fluidized within the suspension.

A common parameter in the pulp and paper industry used to describe the likelihood of floc and/or network formation is the crowding number $N_{C W}$ [34]. The crowding number describes the number of fibres expected in a sphere with a diameter equal to the fibre length:

$$
N_{C W}=5\left[\frac{k g}{m^{3}}\right] \frac{C L_{1}^{2}}{c s} .
$$


Here $C$ denotes the fibre mass concentration in percentage, $L_{1}$ the length-weighted average fibre length, and $c s$ the fibre coarseness (i.e., weight per unit length of fibre). The crowding number was found to describe different fibre interaction regimes: $N_{C W}<1$ : chance of fibrefibre collision, $1<N_{C W}<16$ : dilute fibre flow, $16<N_{C W}<60$ : fibre interaction but no immobilization, and $N_{C W}>60$ : formation of a strong coherent structure with immobilization of fibres. Soszynski and Kerekes [35] performed experiments with straight and smooth nylon fibres in water suspension. They increased fibre concentration, finding non-coherent fibre flocs for $1<N_{C W}<60$, which disappeared again when diluted to $N<1$. Increasing the consistency to reach $N_{C W}>60$, persistent flocs formed, which did not disperse after dilution. Stable coherent flocs formed, and the mechanism was identified to elastic interlocking of fibres. Martinez et. al. [36] showed for fibres settling under gravity, that fibres of different length move freely (i.e., no network effects occur) up to $N_{C W}=16$. For increasing crowding number $N_{C W}$, first the longer fibres became restricted in a network with the shorter fibres still moving freely. At a $N_{C W}$ of 56, all fibres formed a network. The crowding number presenting the first significant change in the system behaviour (i.e., $N_{C W}=16 \pm 4$ ) was termed "gelification point" [36].

Different flow regimes have been identified for floc and network forming fibre suspensions depending on fluid shear stress [19,37]. Specifically, Jäsberg and Kataja [20] identified five regimes for a given pulp (ordered according to the shear stress): (I) plug flow in which fibres are in contact with the wall, (II) plug flow with a thin fluid (water) gap between the wall and the network caused by lift forces, (III) plug flow with a smearing water annulus in which single fibres are disengaged from the plug by turbulent eddies, (IV) mixed flow with a larger water annulus, more intense turbulence, and fibres suspended in the water annulus, and (V) full fluidization of the fibre network. Transition from regime (II) to regime (IV) is sketched in Figure 2. The appearance of the fluid annulus was attributed to lift forces pushing fibres from the wall. 

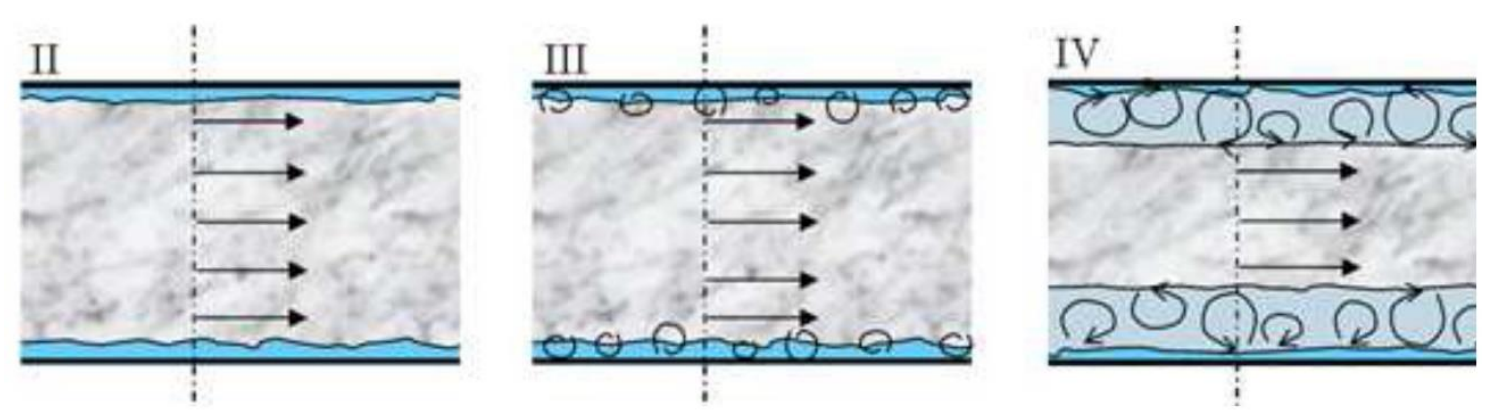

Figure 2: Schematic transition from regime (II) to regime (IV) reproduced from Jäsberg and Kataja [20]. (II) plug flow with a lubrication layer, (III) plug flow with water annulus and turbulent motion in the fluid, (IV) mixed flow with larger water annulus and more intense turbulence.

Norman et al. [19] investigated the stress distribution along the pipe radius. They found, that stress within the plug is caused by fibre interlocking. Its extent depends on the fibre stiffness and the fibre-fibre friction coefficient. Closer to the wall, and for regimes with a fluid annulus, they found contributions due to turbulent (i.e., so-called "Reynolds") stress. Viscous stress contributions dominate very close to the wall. More recently, Nikbakht et al. [38] studied the transition from laminar plug flow to turbulent flow for suspension of differing concentration and fibre length. They found that up to a relative plug size of 0.7 (i.e., the ratio of plug radius to tube radius), fluid stress is smaller than the network yield strength. With increasing size of the water annulus, the fibre network densifies. For increasing fluid stress, exceeding the network yield strength, the plug starts to erode with fibres being torn from the network.

\subsection{Shape and Geometry of the Separation Channel}

The shape of the openings in pressure screens can be divided into (i) screens with holes, i.e. circular separation channel, and (ii) screen with slots, i.e. rectangular separation channel. The screen can either be "smooth", (i.e. a plate with holes or slots), or with contours $[4,10,11,24]$. Contours proved to dramatically improve screen hydraulics, since fluid recirculation vortices within the separation channel block part of the cross section [39]. Introducing contours in the design pulls the vortex from the separation channel into the contour, reducing the hydraulic resistance and improving the screen capacity. Depending on the contour and the resulting shape and size (which also depends on the side channel velocity) of the vortex, larger fibre can be directed into the accept flow. Also, it is argued that contours increase the turbulence level close to the screen, thus helping to disperse fibre networks. Jokinen [12] summarized the effects of screen design showing that contours increase the passage of fibres, but decrease the 
selectivity of the screen. An increase in slot width also increases the passage of fibres, but reduces selectivity.

Tamura et.al. [17] discussed the effect of the orientation of the separation channel for smooth screens. A back-facing separation channel was suggested for separating smaller particles from elongated particles which remain in the reject. The down-stream edge of the separation channel was suggested to have an obtuse angle to increases the entry effect as fibres need to perform greater rotation to enter the side-channel.

\subsection{Recommendations derived from Literature}

Based on the literature on fibre suspension flow, fibre fractionation in pressure screens, and micro fluidics, the following recommendation can be deduced for the design and operation of an HDF:

Separation should be performed at higher concentrations to immobilize longer fibres within a network.

Adjustment of fibre concentration and channel flow rate to establish a water annulus at the wall, whilst taking care that no long fibres are torn from the network and are suspended in the water annulus.

Precise adjustment of the accept flow rate to guarantee the exit layer is located below the fibre plug, i.e., in the water annulus.

Smooth separation channel walls to avoid turbulent motion which would cause the fibre plug to disperse.

$>$ A backfacing separation channel can be advantageous to prevent longer fibres suspended in the water annulus from entering into the accept, and to avoid fibre stapling. 


\section{Materials and Methods}

\subsection{Hydrodynamic Fractionation Device (HDF)}

The proposed HDF is an $885 \mathrm{~mm}$ long channel with a variable rectangular cross section. The channel height can be varied from $3 \mathrm{~mm}$ to $15 \mathrm{~mm}$, and the channel width can be varied from $3 \mathrm{~mm}$ to $9 \mathrm{~mm}$. The top and bottom wall of the channel are made from Plexiglas to allow optical investigation and video analysis. An image of the laboratory HDF and a sketch of the utilities are given in Figure 3.

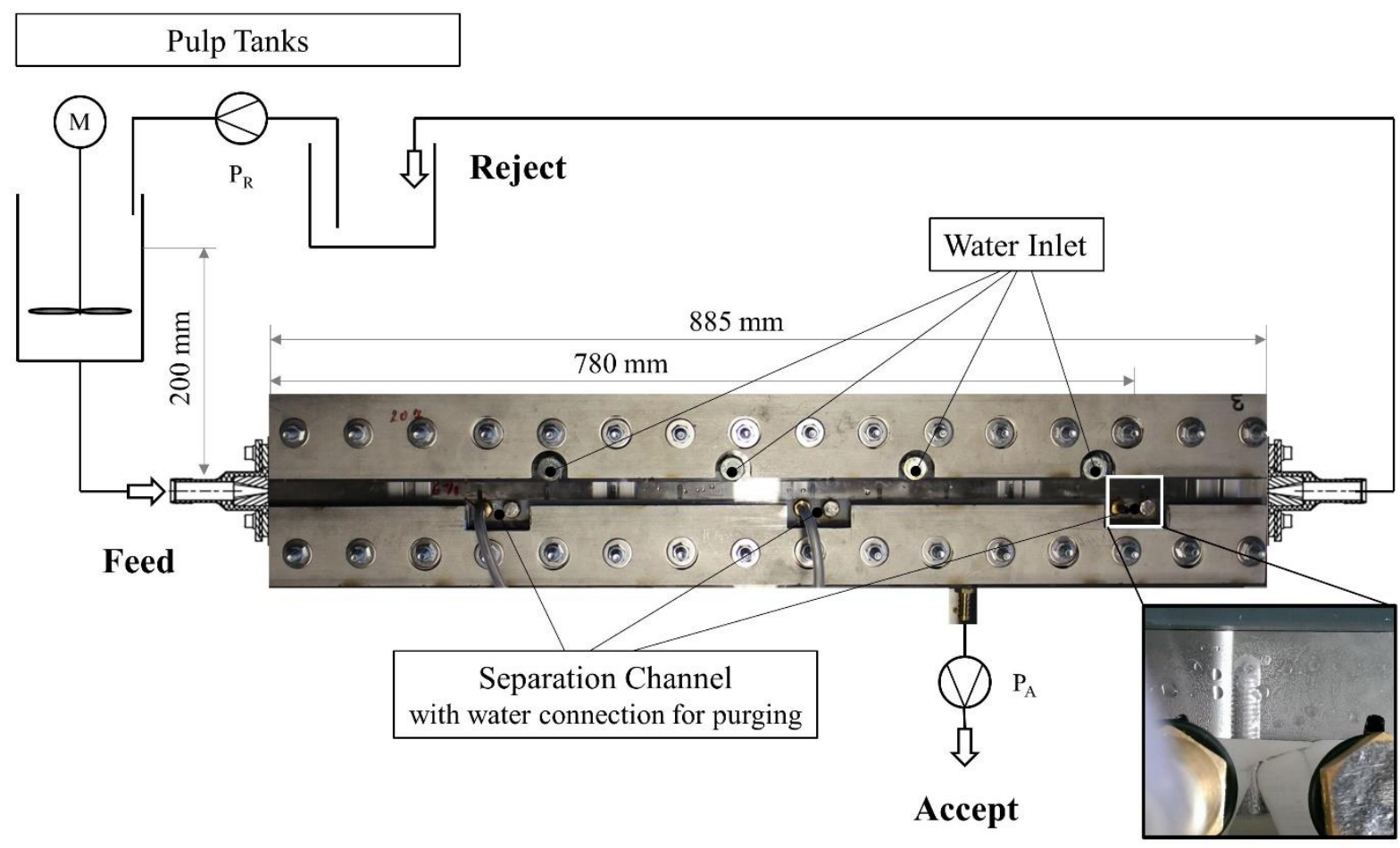

Figure 3: Laboratory HDF with utilities sketched. Utilities comprise of two pulp storage tanks, as well as pumps. Pulp is stirred before feeding to the HDF. The suspension level is $200 \mathbf{~ m m}$ above the HDF inlet. The third separation channel (white box) is presented in a close up showing the two connections for the water purge on the left and right of the close-up. The accept flow is set with pump $P_{A}$.

The laboratory HDF is designed with three separation channels, and a water inlet for dilution of the suspension on the opposite side. In the first experimental investigation, reported in our present contribution, separation was only performed with the third separation channel, positioned $780 \mathrm{~mm}$ downstream of the channel inlet. The HDF was designed for fast and easy switching of the separation channel, which was produced via additive manufacturing. 
Fibre suspension was supplied from a feed tank (approx. $10 \mathrm{~L}$ ) which was connected via a feed pipe ( $D=18 \mathrm{~mm}$ ) to the HDF. The connectors (inlet and outlet) for the tube and HDF were produced by additive manufacturing and customized for a specific channel cross section. The accept flow rate was set using a peristaltic pump $\mathrm{P}_{\mathrm{A}}$ (Ismatec ECOLINE VC-280/281, Cole-Parmer GmbH, Germany). Thus, the actual flow oscillated (insignificantly) around the given mean flow rate. The main channel flow rate was set by applying a static pressure difference between the suspension in the feed tank, and the tube connected to the exit of the HDF. The suspension height in the feed tank was set by an overflow. The flow rate was determined by weighting samples collected over periods of $20 \mathrm{~s}$ to $30 \mathrm{~s}$. The reject was collected and pumped back into the feed tank $\mathrm{P}_{\mathrm{R}}$ (Ponndorf, Hennlich $\mathrm{GmbH}$, Austria). The total amount of removed fines and water during the experiment was low enough to ensure a very slow temporal change of the pulp composition in the tank. The feed flow rate differed slightly between different experiments with same settings. .

The side channel geometry is crucial for the separation process as it (i) impacts the flow pattern, (ii) defines fibre movement including fibre stapling at the exit, and (iii) contributes to the pressure loss. Based on the literature review, two geometries were tested, differing in the orientation of the separation channel. These were arranged under and angle of $90^{\circ}$, and $20^{\circ}$ in relation to the upstream wall. Drawings of the separation channel and the manufactured channels are shown in Figure 4.
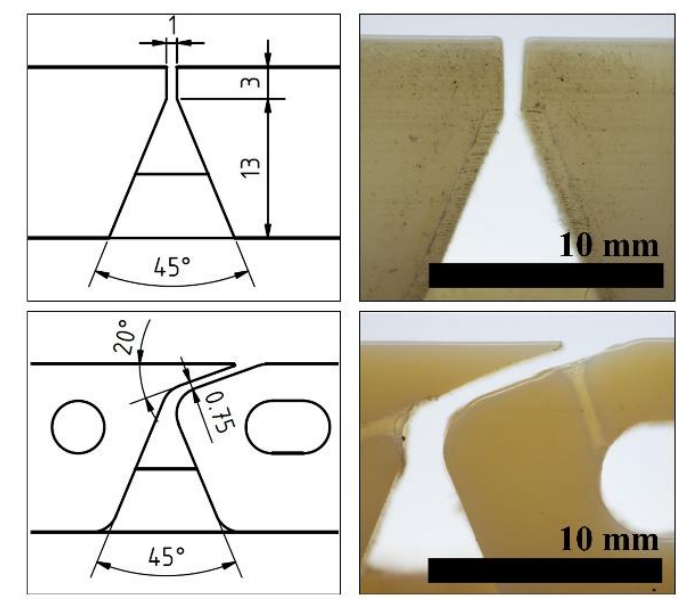

Figure 4: Shop drawings and pictures of the manufactured separation channels. Top panels: $90^{\circ}$ separation channel, with opening of $1 \mathrm{~mm}$. Sharp corner at the downstream edge and a diverging collection channel. Bottom panel: $20^{\circ}$ separation channel, with channel width of $0.75 \mathrm{~mm}$. Two different purging positions are realized. Purging channels are visible in the picture as lighter spots. 
The channel width was $1 \mathrm{~mm}$ for the $90^{\circ}$ channel, and $0.75 \mathrm{~mm}$ for the $20^{\circ}$ channel. Both channels were diverging to reduce pressure drop in the separation channel and reduce the risk of blockage. Purging channels were added to ensure fast removal of stapled fibres. The connection for the purge water is drawn in the shop drawing, i.e., Figure 4, bottom panel. Preliminary tests showed larger efficiency of the left purge channel (having a circular connection in the shop drawing shown in Figure 4). The other purge channel was sealed. The purge time was always $0.05 \mathrm{~s}$, and the pause time between purges was adjusted and differed for various experimental cases. The pause time ranged from $2.5 \mathrm{~s}$ to $180 \mathrm{~s}$. The operational performance of both channels was investigated in preliminary experiments and their results are discussed in Chapter 4.1.

\subsection{Pulp Material}

Experiments had been performed with chemical pulp provided by Sappi Gratkorn (Austria). The chemical pulp was of $100 \%$ spruce, bleached and washed, and provided at a concentration of $17 \%$. The pulp was diluted to fibre concentrations of $0.1 \%$ and $0.5 \%$ for the experiments. Higher concentration lead to blockage of the channel inlet, at the contraction in the current setup. Pulp properties were determined from three samples with a L\&W Fiber Tester $+($ Lorentzen + Wettre, Sweden $)$ and are listed in Table 1. The crowding number $N_{C W}$, was calculated using equation 1 .

Table 1: Pulp properties. Charactersistic mean fibre length $L_{1}$, and $L_{3}$ and fibre coarseness $c s$. Crowding number $N_{C W}$ for $0.1 \%$ and $0.5 \%$ fibre concentration according to eq. 1 .

\begin{tabular}{lcc}
\hline Length-weighted fibre Length $L_{1}$ & $1.820 \mathrm{~mm}$ \\
Volume-weighted fibre Length $L_{3}$ & $2.447 \mathrm{~mm}$ \\
Fibre coarseness cs & $0.174 \mathrm{~g} / \mathrm{m}$ \\
\hline Crowding number $N_{C W}$ for $C 0.1 \%$ & 9.5 \\
Crowding number $N_{C W}$ for $C 0.5 \%$ & 47.5 \\
\hline
\end{tabular}




\subsection{Measurement Procedure}

Samples were taken from the feed suspension (initial pulp) and the accept flow. The feed suspension was sampled at the channel exit stopping the fractionation process, i.e., setting the accept flow rate to zero. The experiment was run for several channel flow-through times before sampling the feed suspension and after re-starting the fractionation process. Sampling was performed three times for all the experimental settings.

The fibre concentration $C$ in the samples was determined by means of thermogravimetric weighting. The fibre length distribution $\Delta Q_{3}$, the length-weighted $L_{1}$, and volume-weighted $L_{3}$ mean fibre length of the samples were determined with a L\&W Fiber Tester + (Lorentzen + Wettre, Sweden) according to ISO 16065-2:2014 standard. Fibre length classes for the evaluation were defined as $[0.0,0.2,0.6,1.0,2.0,3.0,4.0,5.5] \mathrm{mm}$.

The length-selective separation process was quantified by the grade efficiency $T(x)$. The grade efficiency $T(x)$ states the fraction of the feed found in the coarse fraction (reject flow) per fibre class $(x)$. Related on the fines fraction (accept flow), the grade efficiency $T(x)$ was calculated as:

$$
T(x)=1-\frac{\dot{m}_{\text {fine }}(x)}{\dot{m}_{\text {feed }}(x)}=1-\frac{\dot{m}_{\text {fine }} \Delta Q_{3, \text { fine }}(x)}{\dot{m}_{\text {feed }} \Delta Q_{3, \text { feed }}(x)} .
$$

$\dot{m}$, and $\dot{m}(x)$ state the mass flow and the mass flow per size class, respectively. $\Delta Q_{3}$ is the mass or volume based distribution per size class. Evaluating the length-based separation of the HDF, the grade efficiency $T(x)$ depends on the mean fibre length per class $i$, thus $T\left(l_{f i b r e, i}\right)$. The density of the fibre can be assumed equal for all length fractions. Thus the grade efficiency for the process can be formulated as:

$$
T\left(l_{\text {fibre }}\right)=1-\frac{\dot{V}_{\text {accept }} C_{\text {accept }} \Delta Q_{\text {accept }}\left(l_{\text {fibre }}\right)}{\dot{V}_{\text {feed }} C_{\text {feed }} \Delta Q_{\text {feed }}\left(l_{\text {fibre }}\right)} .
$$

The grade efficiency $T\left(l_{\text {fibre }}\right)$ was determined for every experiment, and we report the mean and standard deviation per class in what follows.. 
The separation efficiency based on the fibre mass in the accept stream $f$ is calculated from the grade efficiency to yield a single value for comparing different cases. $f$ states the mass fraction of fibres from the feed found in the accept flow:

$$
f=\frac{\sum \dot{V}_{\text {accept }} C_{\text {accept }} \Delta Q_{\text {accept }}\left(l_{\text {fibre }}\right)}{\sum \dot{V}_{\text {feed }} C_{\text {feed }} \Delta Q_{\text {feed }}\left(l_{\text {fibre }}\right)}
$$

Images of the fibre suspension flow at the side channel were recorded at a frequency of $3000 \mathrm{~Hz}$ (IDT Os8-S3, resolution of 1600x1200 pixel, Imaging Solution GmbH, Germany).

\subsection{Experimental Plan and Settings}

The $90^{\circ}$ and $20^{\circ}$ separation channel geometry were tested in preliminary experiments. The influence of the operation parameters $\left(R e, \Phi^{+}\right.$, and $\left.C\right)$, and design parameters $(H, W)$ were tested as variation of a base case. Case settings are highlighted in Figure 5. A comprehensive list of all experimental settings and case ID is given in the Appendix, Table 2. Experiments were aimed to be performed at two different Reynolds number, i.e., $R e$ 1300, and $\operatorname{Re} 4000$, and two different feed concentrations $C_{\text {feed }} 0.1$ and $C_{\text {feed }}$ 0.5. Slight fluctuations of the flow rate around the documented mean value were noticed. Likewise, the dimensional accept flow rate $\Phi^{+}$deviated slightly from desired value. The actual value was recorded and stated with every experiment (see Appendix).

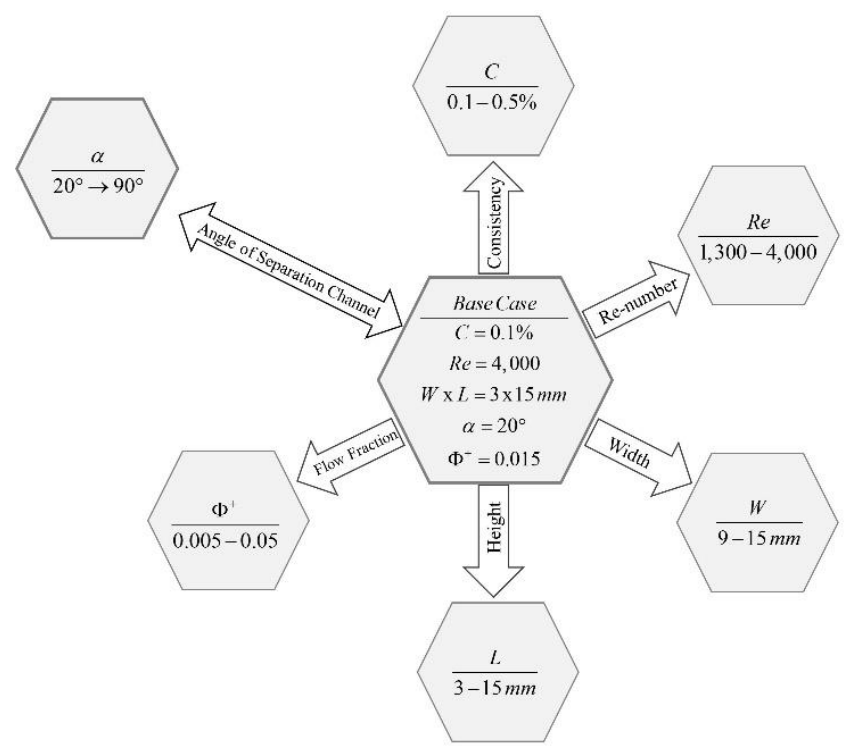

Figure 5: Variation star highlighting the range of settings for different experimental trials. 


\section{Results and Discussion of the Length-Based Separation}

Results for every case, i.e., fines separation efficiency $f$, and fibre length mean $L_{1}$, and $L_{3}$ per case are listed in the Appendix, Table 3. The general trend was a low fines separation efficiency using only one channel, but a high selectivity. This means that the HDF shows excellent performance in terms of a sharp separation. The results are discussed in greater detail in the following sub-chapters.

\subsection{Effect of the Separation Channel Shape}

Preliminary separation experiments were performed with fibre suspensions at a concentration $C_{\text {feed }}$ of approx. $0.1 \%$, and a relative accept flow rate $\Phi^{+}$of 0.015 . The $90^{\circ}$ separation channel and $20^{\circ}$ separation channel (see Figure 4) were tested. The experiments were performed at $R e$ 1300, and $R e$ 4000. The side channel was purged to prevent blockage, and the purge interval was adjusted to allow constant operation at the lowest possible purge interval.

The results for the grade efficiency (Figure 6) for both geometries were similar and did not differ significantly. The mean fibre length $L_{1}$, and $L_{3}$, as well as the separation efficiency $f$ were also similar. Both geometries were thus found to perform equally in terms of separation performance at tested operational parameters. These findings differ from previously reported findings for stiff fibres: in those cases, a lower acceptance was reported with a backfacing separation channel [17]. The flexible fibres used in our present study, however, are expected to form a network. Thus, our results suggest that only smaller fibres were suspended in the annulus, and removed at almost identical rates by both separation channel geometries.

The rate at which fibres stapled at the downstream edge of the separation channel differed. Long fibres accumulated at the downstream edge of the separation channel entry for the $90^{\circ}$ geometry (see Figure 7, left image), but were swept over the entry for the $20^{\circ}$ geometry (see Figure 7 , right image). To prevent the $90^{\circ}$ separation channel from fully blocking, the channel had to be purged every $2.5 \mathrm{~s}$ for $\operatorname{Re} 4000$, and every $20 \mathrm{~s}$ for $\operatorname{Re} 1300$. The obtuse angle at the downstream edge of the $20^{\circ}$ separation channel reduces the tendency for fibre stapling significantly. Long fibres turned and moved when coming into contact with the wall or the 
edge, and had been re-entrained in the suspension flow at a higher rate. Continuous operation with the $20^{\circ}$ separation channel was possible at purge intervals of $60 \mathrm{~s}$ and longer.

Thus, consecutive separation experiments were performed with the $20^{\circ}$ separation channel due to its superior operability.

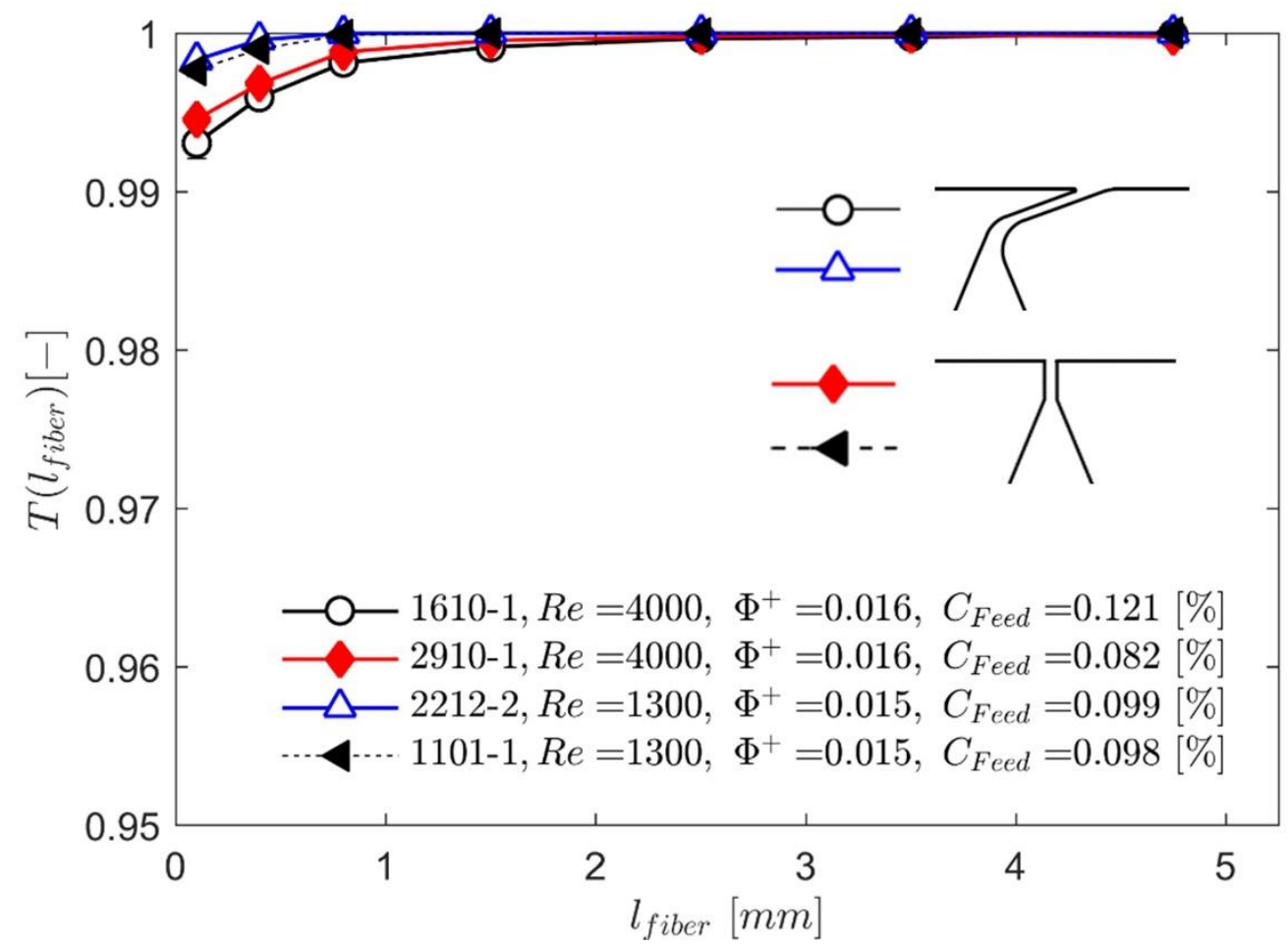

Figure 6: Grade efficiency for $90^{\circ}$ (filled marker) and $20^{\circ}$ (open marker) separation channel. Fibre concentration approximately $0.1 \%$, and relative accept flow of 0.015 . The Reynolds number was varied between 4000 and 1300 .
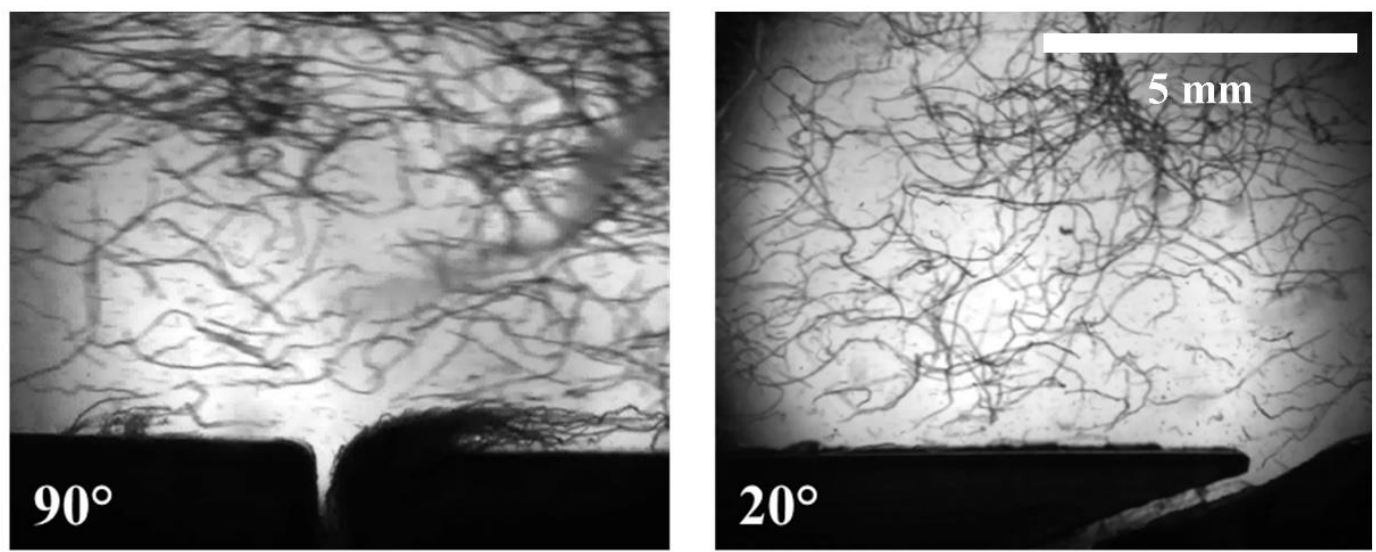

Figure 7: Fibre suspension flow over the $90^{\circ}$ separation channel (left panel), and $20^{\circ}$ separation channel (right panel). $R e$ 4000, fibre concentration $C_{\text {feed }} 0.1 \%$, and relative accept flow rate $\Phi^{+} 0.015$. 


\subsection{Reynolds Number}

The Reynolds number $R e$ was varied between 4000 and 1300. The experiments were performed at high and low accept flow rate ( $\Phi^{+}$of approx. 0.05, case 1301-1, and 2710-1, and $\Phi^{+}$of approx. 0.015, case 1610-1, and 2212-2). Grade efficiency results are presented in Figure 8. At low $\Phi^{+}$, an increase in $R e$ lead to an increase in the removal of fibres smaller than $1 \mathrm{~mm}$. The removal of larger fibres was negligible for both cases. Consequently, the mean fibre length and fines separation efficiency $f$ increased with $R e$. At higher accept flow rate $\Phi^{+}$and low $R e$ a good separation of fibres $<1 \mathrm{~mm}$ was achieved. Increasing $R e$ led to the removal of long fibres with the accept flow. The resulting mean fibre length $L_{1}$, and $L_{3}$ was well above $1 \mathrm{~mm}$, yielding the worst separation efficiency of all tested cases. Consequently, the fines separation efficiency $f$ was comparably high (i.e., 1.76\%). The combination of high $\Phi^{+}$, and high $R e$ results in the largest observed accept flow rate.

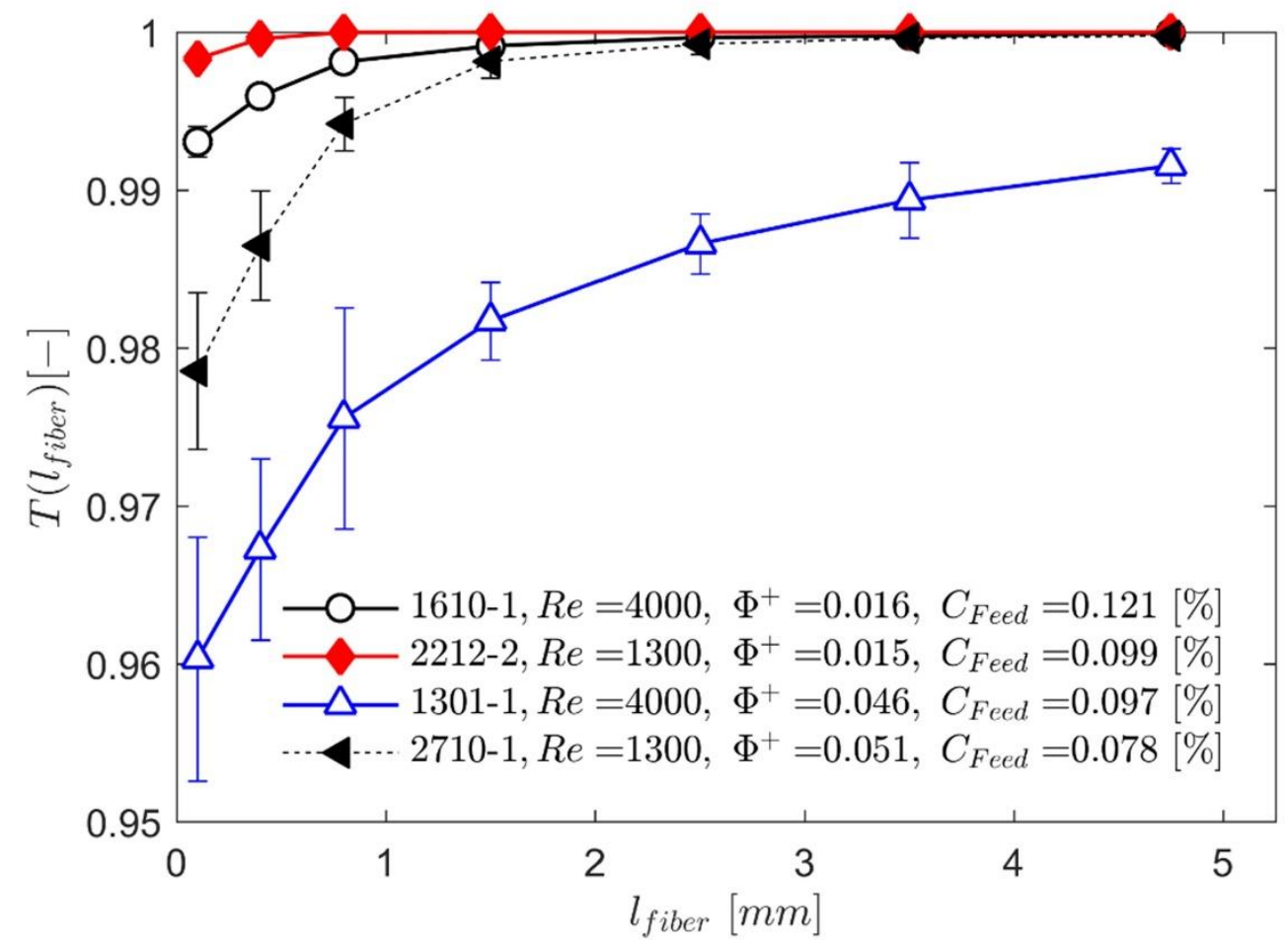

Figure 8: Grade efficiency for differing $R e$ and $\Phi^{+}$in $3 \times 15$ mm channel.

High-speed images were recorded, and representative snapshots for $\operatorname{Re} 1300$ and $\operatorname{Re} 4000$ are presented in Figure 9. The corresponding animation is provided in the electronical Annex. The white double line indicates the interface between the fibre network plug and the water annulus. The interface was relatively sharp for $R e 1300$, but diffuse for $\operatorname{Re} 4000$. The network 
extended over the full channel height for $R e$ 4000, however fibres were not fully fluidized for this flow condition. The black dashed, and the black dash-dotted line are estimates of the mean exit layer corresponding to $\Phi^{+}$of approximately 0.050 and 0.015 , respectively. Since the accept flow rate was slightly pulsating, the presented estimate of the exit layer offers only a qualitative guide at which location the separation occurred.
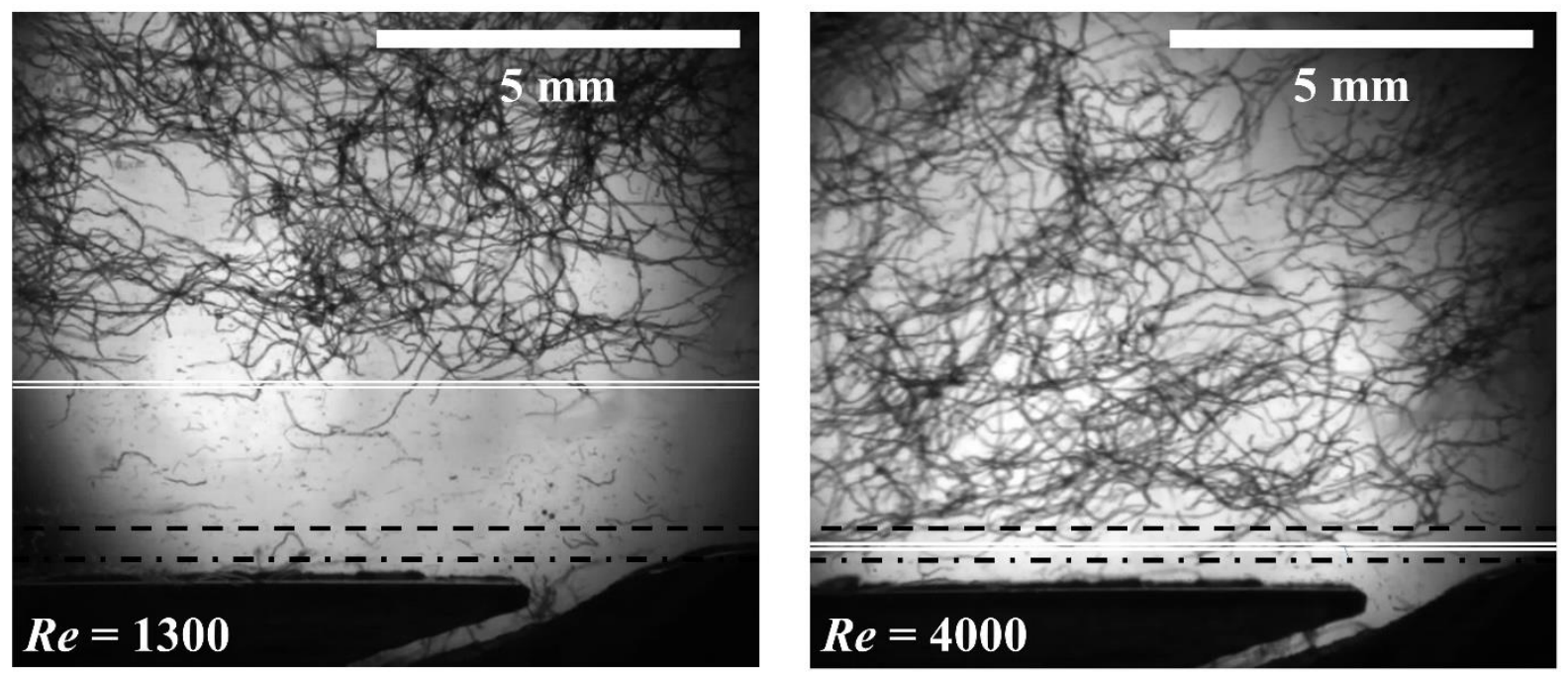

Figure 9: Images of the junction of the main and the separation channel. Left image: $R e$ 1300, right image $R e$ 4000. The mean fibre concentration of the feed suspension $C_{f e e d}$ is $0.1 \%$. The crowding number $N_{C W}$ is 9.5. The fibre network extends over the full channel height for larger $R e$, and is confined to a region near the channel center for lower $R e$.

Specifically, we found the exit layers well below the plug interface for $R e$ 1300. Fibre fines and smaller fibres suspended in the water annulus were removed with the accept flow. The looser network for Re 4000 lead to a larger extend of the network over the channel height. The exit layer was estimated above the network interface for higher accept flow rate. For lower accept flow rate, we find the exit layer below the interface, with some longer fibres suspended below the interface. We argue, that those long fibres were removed at a lower rate due to the wall and turning effect discussed in Chapter 2.1. Comparing our findings to literature results, the theory of fibre separation for dilute suspension was to some degree applicable for high $\operatorname{Re}$ flow (i.e., $\operatorname{Re} 4000$ ). For low $R e$ and a fibre suspension flow within the annulus plug flow regime, fibre immobilisation by floc formation was identified as the root cause for the observed length-based separation.

This clearly highlights the importance of the side channel flow rate to achieve optimal fractionation results: the position of the exit layer relative to the fibre network interface is influenced by the side channel flow rate, and is hence investigated next. 


\subsection{Side Channel Flow Rate}

The dependency of the grade efficiency $T(x)$ on the accept flow rate was studied in greater detail for the higher flow rate, $R e$ 4000. With respect to the $R e$ number study, experiments were performed at lower accept flow rates, ranging from $\Phi^{+}=0.005$ to $\Phi^{+}=0.046$. The corresponding grade efficiency results are presented in Figure 10.

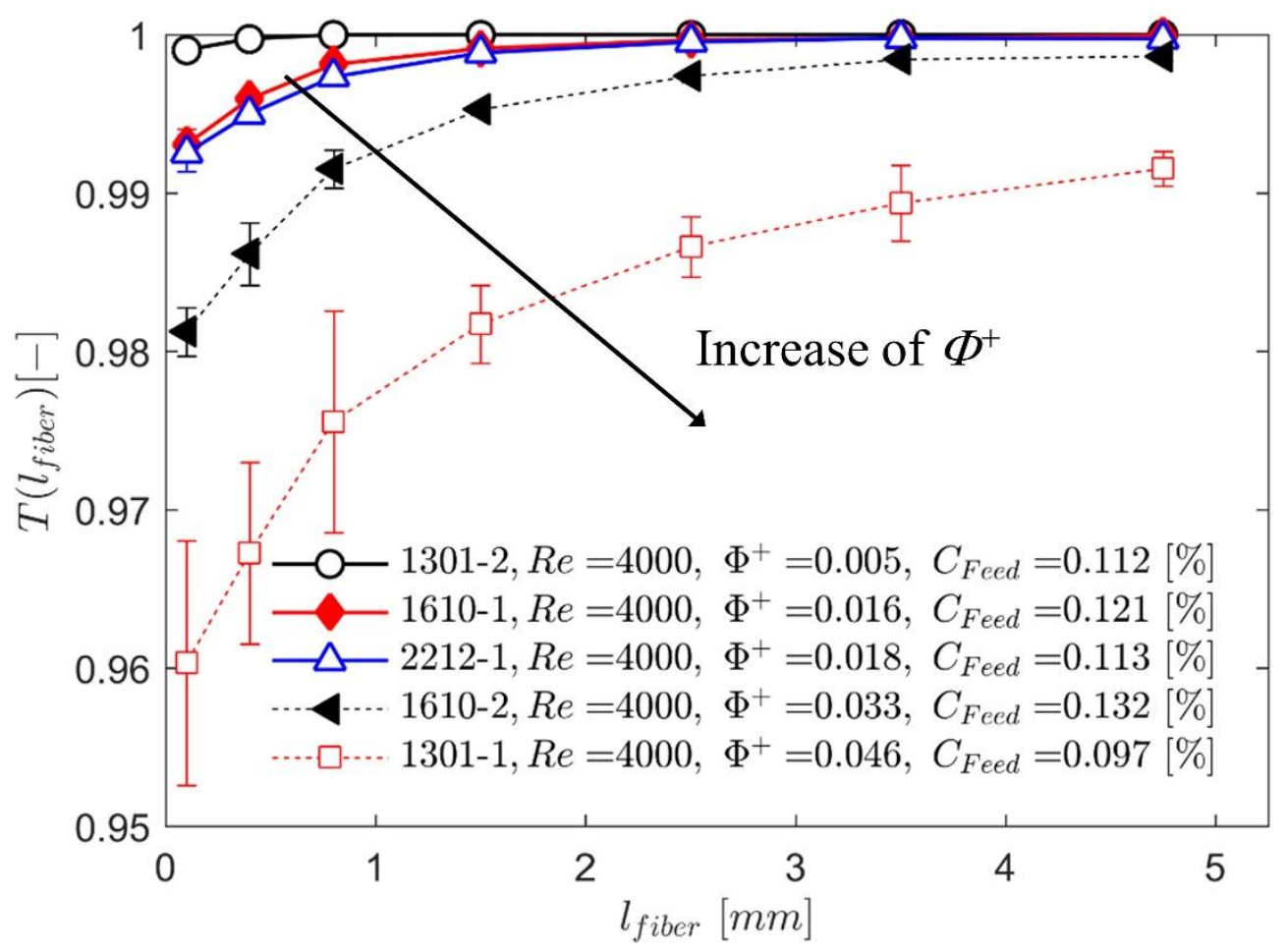

Figure 10: Grade efficiency for $\Phi^{+}$from 0.005 to 0.046 in $3 \times 15 \mathrm{~mm}$ channel at $R e 4000$.

For $\Phi^{+}$up to approx. 0.02 good separation of fibres $<1 \mathrm{~mm}$ was achieved. For the case with the lowest side channel flow rate (i.e., experiment $1301-2, \Phi^{+}=0.005$ ) the grade efficiency was similar to case 1610-1 (Figure 8). Also, similar mean fibre lengths were measured. However, the separation efficiency of experiment 1301-2 (i.e., $f=0.012$ ) was only half of experiment $1610-1$ (i.e., $f=0.023$ ) at a comparable absolute accept flow rate. Increasing $\Phi^{+}$to 0.033 led to an unwanted removal of long fibres with the accept flow. Doubling the accept flow rate increased the number of removed shorter and longer fibres. The grade efficiency was lower, however, the shape of the curve for the two latter cases was similar.

The results from the detailed study on the influence of the accept flow rate $\Phi^{+}$provide an addition to the previously reported findings for relatively dispersed fibre suspension flow. At low $\Phi^{+}$the exit layer was close to the wall, such that longer fibres were retained from 
removal caused by the wall-effect and/or turning effect. In contrast, at higher $\Phi^{+}$longer fibres were suspended within the exit layer, and could turn when approaching the side channel. Thus, they were removed with the accept flow. Further increase of the accept flow rate led to larger removal of fluid and the suspended fibres. In summary, and based on our observations of fibre behaviour in the channel, we hypothesize that only freely moving fibres below the exit layer were removed. However, fibres linked to the network remained in the reject.

\subsection{Consistency}

The maximum fibre concentration for the current setting of the HDF was $C_{\text {feed }}$ of $0.5 \%$ for $R e$ of 4000. Above that concentration, and/or at lower Reynolds number Re, inlet and outlet of the main channel were prone to plugging. Thus, experiments at higher consistency were only performed for $R e 4000$ (case 0510-1, and 0510-2). The results were compared to the corresponding cases at lower concentration $C_{\text {feed }}$ of approximately 0.1 , and the resulting data for the grade efficiency is presented in Figure 11.

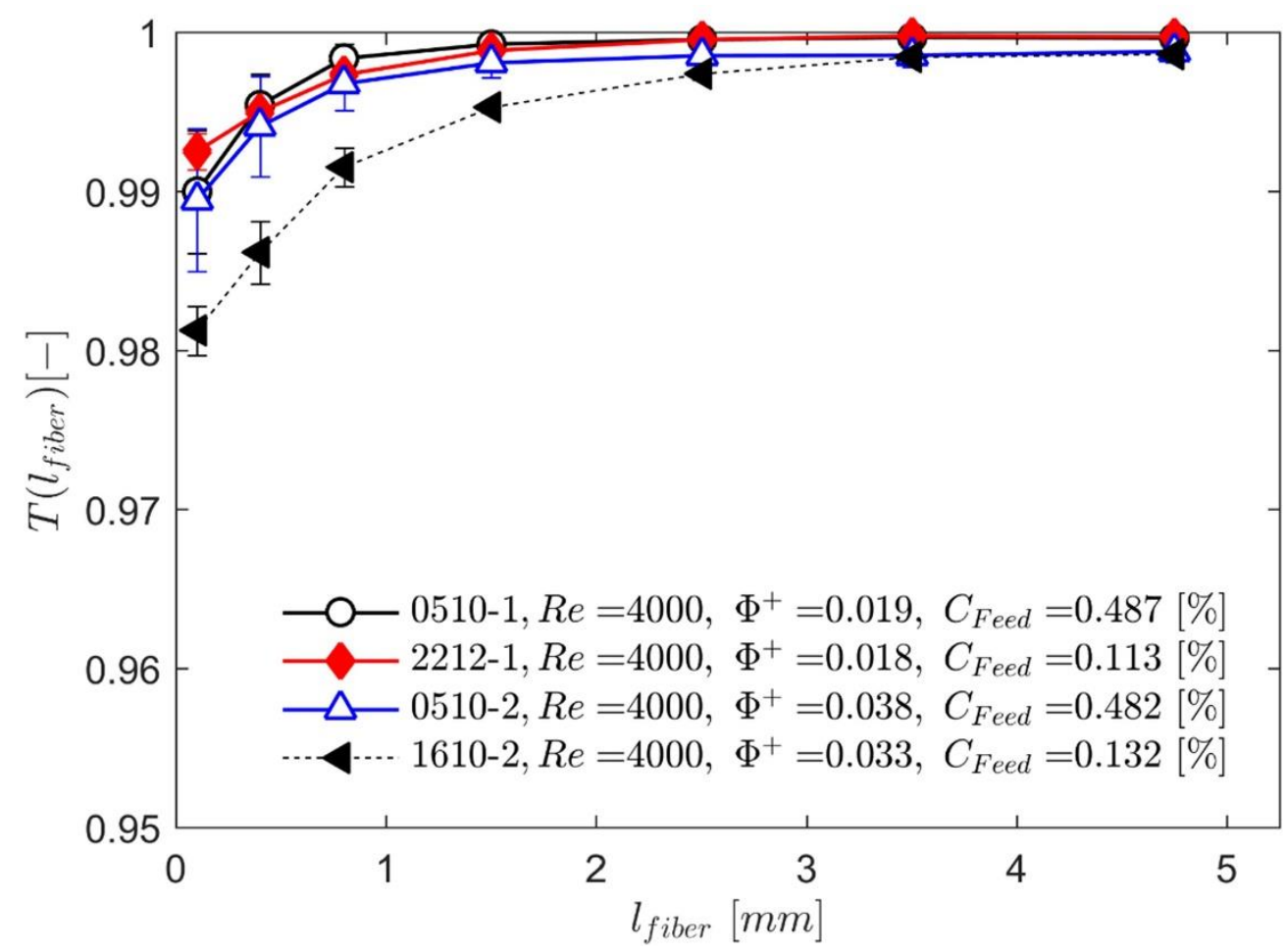

Figure 11: Grade efficiency for $C_{\text {feed }}$ being approx. $0.1 \%$, and $0.5 \%$ in $3 \times 15 \mathrm{~mm}$ channel at $R e$ 4000, and $\Phi^{+}$of approx. 0.15 , and ca. 0.4 .

At low $\Phi^{+}$we found no differences in the grade efficiency when changing the fibre concentration. Likewise, mean fibre length and separation efficiency were similar. Following 
our previous observations, we expect longer fibres being above the exit layer and thus not removed with the accept flow. At higher $\Phi^{+}$, we found the grade efficiency lower for smaller fibres and removal of longer fibres, i.e., sub-optimal fractionation. As the grade efficiency states the separation relative to the feed, the concentration of fibres in the accept increases with the feed concentration for constant grade efficiency values. This means that a larger number of long fibres is removed when operating the HDF with a suspension concentration $C_{\text {feed }}$ of $0.5 \%$.

We argued above that at higher $\Phi^{+}$longer fibres which were fluidized in the near-wall suspension were removed with the accept flow. At increasing fibre concentration the number of fibre-fibre contacts increases. For given pulp properties (see Table 1) the crowding number $N_{C W}$ increased from 9.5 for case $1610-2$ to 47.5 for case $0510-2$. Thus, the network of fibres could be expected to be stronger. We hypothesize that smaller fibres ( $1 \mathrm{~mm}$ to $3 \mathrm{~mm}$ ) were linked more tightly into the fibre network, and hence the removal with the accept stream dropped. Our hypothesis follows findings of a reduced fibre mobility with increasing crowding number reported by Martinez et.al. [36]. For settling fibres, this previous study found that smaller fibres were networked once the crowding number $N_{C W}$ exceeded a threshold of $16 \pm 4$. However, our hypothesis could not be further assessed in our present study, and is subject to ongoing research activities.

\subsection{Channel Height}

The channel height $H$ was reduced from $15 \mathrm{~mm}$, to $9 \mathrm{~mm}$, and to $3 \mathrm{~mm}$. The channel width remained at $3 \mathrm{~mm}$. Thus the suspension flow in the main channel was affected, but not the accept flow in the side channel as the geometry was unchanged. Separation experiments were performed with a suspension of approx. $0.1 \%$ fibre concentration $C_{f e e d}$ at Reynolds numbers $R e$ 1300, and $R e$ 4000. Performing experiments at $H$ of $3 \mathrm{~mm}$ led to channel plugging in short time, and thus operating the HDF was not possible. Consequently, the separation performance was evaluated for cases of $H 15 \mathrm{~mm}$, and $9 \mathrm{~mm}$ only. Grade efficiency results are presented in Figure 12. Images of the suspension flow were recorded and are shown in Figure 13.

Due to lower limits of the accept flow by the pump, experiment 1612-1 (H $9 \mathrm{~mm}$, and $R e$ 1300) was performed at $\Phi^{+}$being $50 \%$ higher compared to the other cases. Thus the results 
might also have been affected by the change in the accept flow rate. No significant effect of the channel height on the grade efficiency was found for high and low $R e$.

The interface between the fibre network and the water annulus was estimated from image analysis, indicated as a white double line in Figure 13. Image analysis showed a smaller water annulus for $R e 1300$ case at lower channel height $H$ of $9 \mathrm{~mm}$. At low accept flow rate the exit layer was well below for the interface in both cases. Consequently the grade efficiency was similar. At higher $R e$ we found the water annulus smaller for both channel heights, what corresponds to the previous reported results on the effect of the Reynolds number. Very little difference was found between the two different channel heights judging from image analysis (see Figure 13 bottom panel). As argued above, at low accept flow rate fibres were retained from removal due the wall and turning effect.

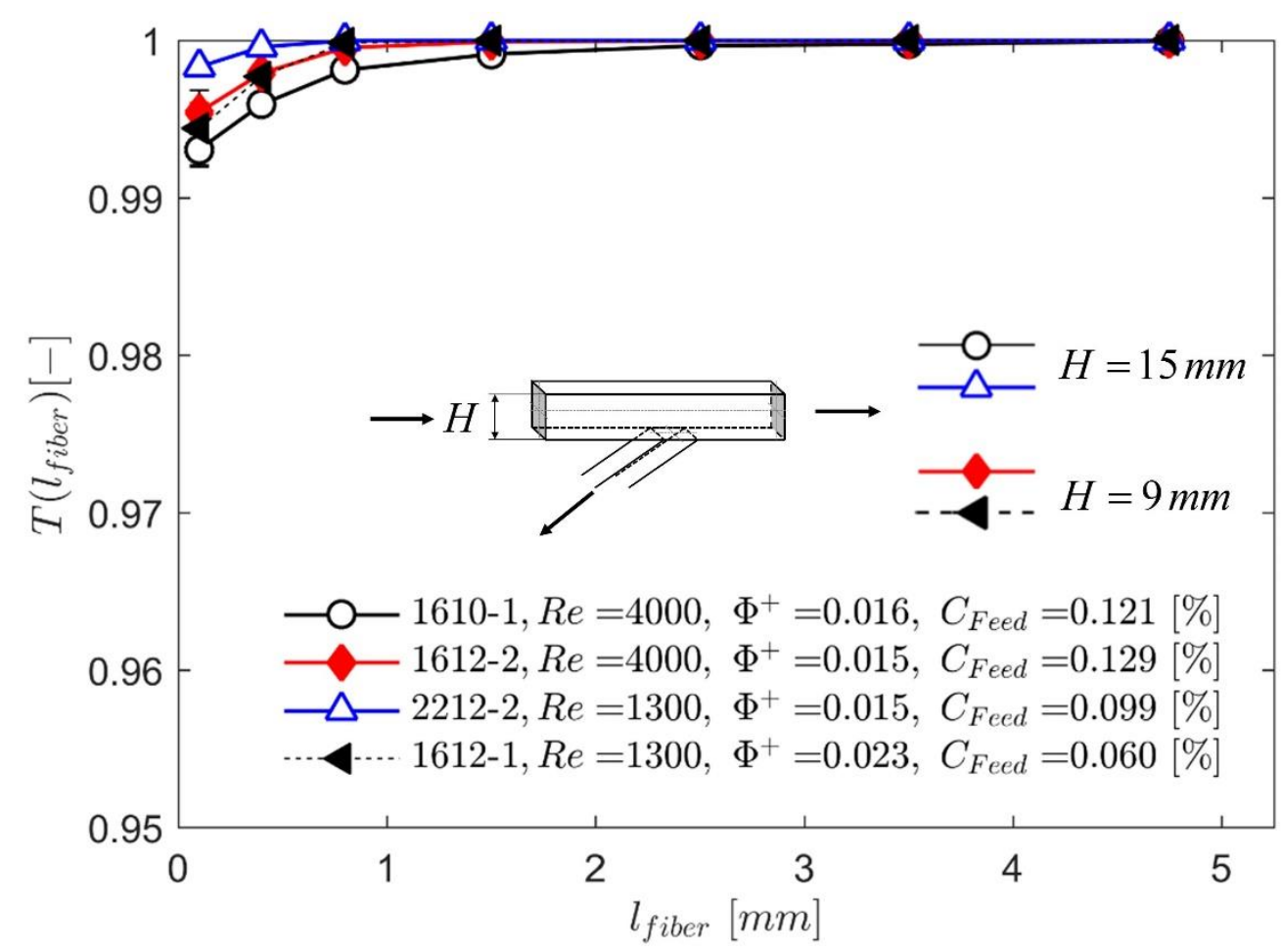

Figure 12: Grade efficiency in dependence of the channel height $\boldsymbol{H}$ for $\boldsymbol{C}_{\text {feed }}$ of approx. $0.1 \%$, channel width $W$ of $3 \mathrm{~mm}, \operatorname{Re} 4000$, and $\Phi^{+}$of approx. 0.15 . 


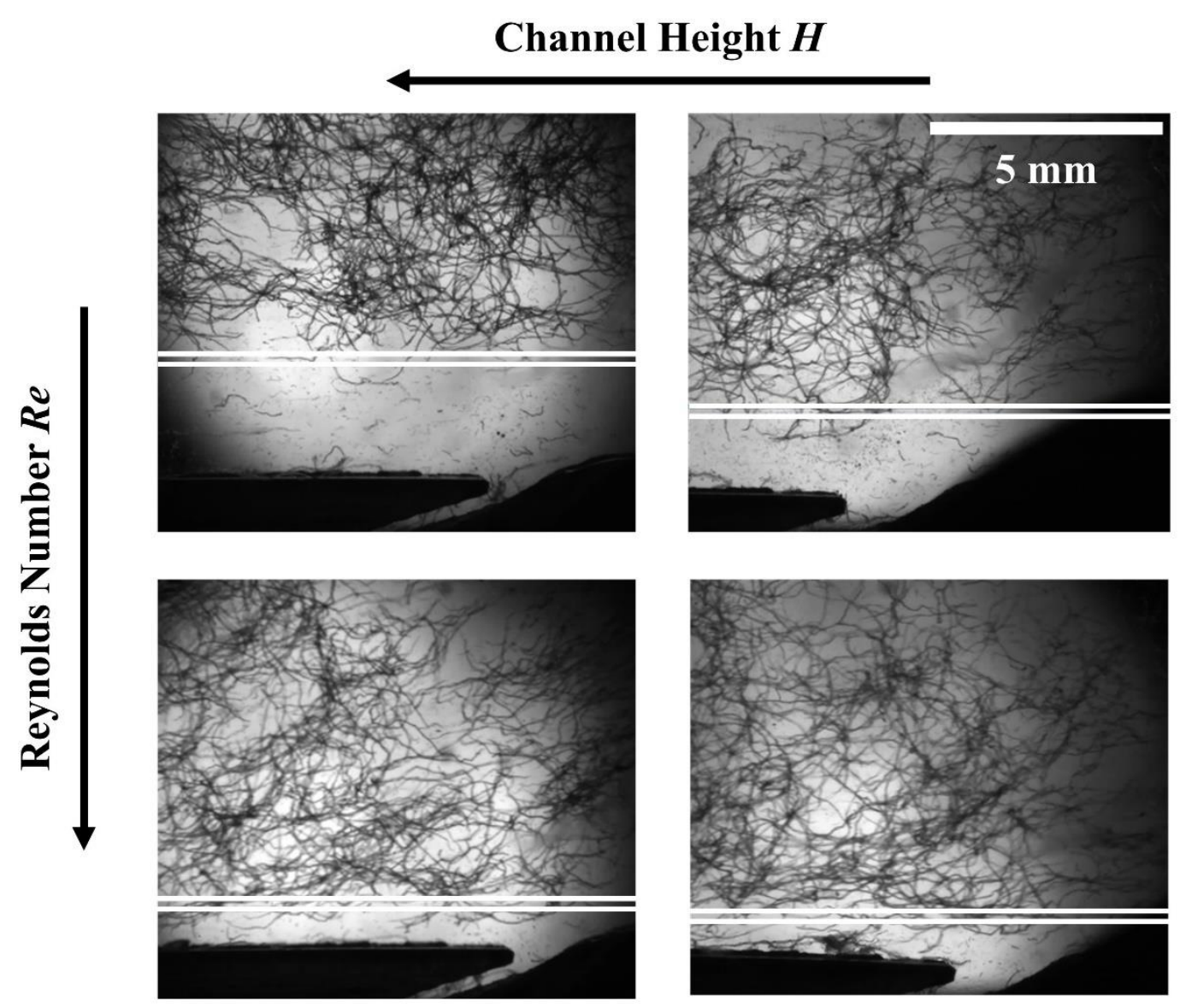

Figure 13: Images of the main channel at the position of the separation channel. Upper images: Re 1300, lower images: $R e$ 4000. Left images: $H$ of $9 \mathrm{~mm}$, right images: $H$ of $15 \mathrm{~mm}$. Fibre concentration $C_{\text {feed }}$ of $0.1 \%$, resulting in a crowding number $N_{C W}$ of 9.5 . White double lines indicate the estimated interface between fibre network and the water annulus.

\subsection{Channel Width W}

The channel width $W$ was increased from $3 \mathrm{~mm}$ to $9 \mathrm{~mm}$ for the standard height of $H$ of $15 \mathrm{~mm}$. Experiments were performed with $C_{\text {feed }}$ of approx. $0.1 \%, \Phi^{+}$of approx. 0.015 , and at $\operatorname{Re} 1300$ and $\operatorname{Re} 4000$. The resulting data for the grade efficiency is presented in Figure 14.

Increasing the channel width $W$ also affected the cross section of the separation channel, i.e., it was increased by a factor of three. Thus, a larger absolute accept flow rate could be achieved. The shape of the separation channel changed from small slots (with a cross section of $3 \mathrm{~mm}$ by $1 \mathrm{~mm}$ ) to more elongated slots (i.e., a cross section of $9 \mathrm{~mm}$ by $1 \mathrm{~mm}$ ). No significant difference in the grade efficiency was found for increasing the channel width from $3 \mathrm{~mm}$ to $9 \mathrm{~mm}$ when increasing the Reynolds number $R e$. 
Manuscript - Length-Selective Separation of Cellulose Fibres by HDF

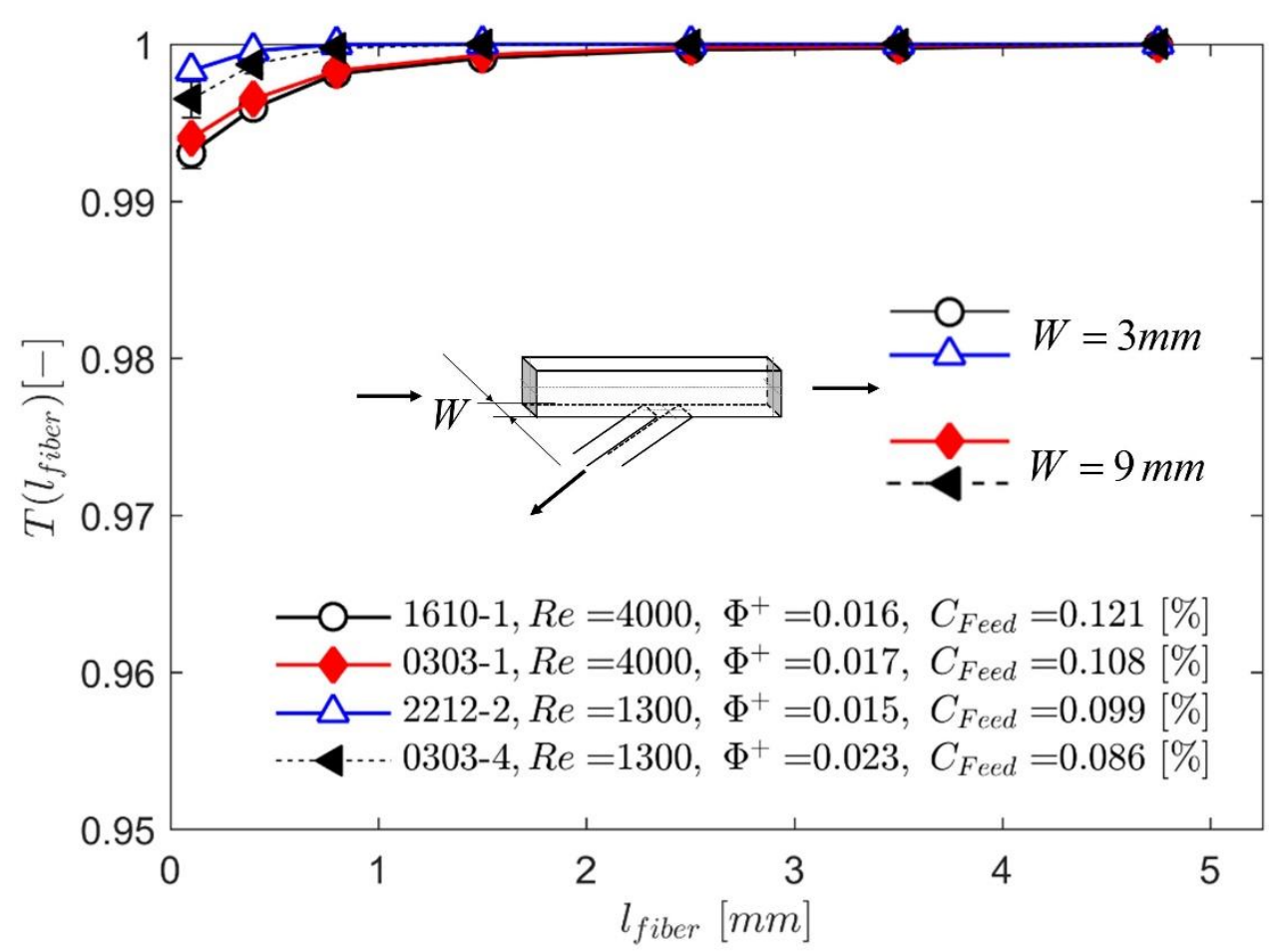

Figure 14: Grade efficiency in dependence of the channel width $W$ for $C_{\text {feed }}$ of approx. $0.1 \%$, channel height $H$ of $15 \mathrm{~mm}, \operatorname{Re} 4000$, and $\Phi^{+}$of approx. 0.15. 


\section{Conclusion}

The hydrodynamic fractionation device HDF presents a new type of fractionator for length based fibre separation. The fractionation principle is based on (i) the effective retention of long fibres in fibre flocs, as well as (ii) the removal of smaller fibres suspended in a wallbounded fluid layer. While previous literature identified relevant flow regimes that affect the fractionation principle of HDF, we have explored the effect of Reynolds number $R e$, and network strength (which is influenced by the concentration for a given type of cellulose pulp) on the separation efficiency of the HDF.

Specifically, the HDF was tested with a fibre suspension at a concentration of $0.1 \%$ yielding a moderate network strength expressed by the crowding number $N_{C W}$ of 9.5. The separation process was evaluated in terms of grade efficiency $T(x)$ which states the relative amount of fibres or fines that exits as reject. Variation of the channel geometry, the channel height and the channel width did not significantly affect the grade efficiency within tested parameter space. A substantial effect on the separation was found for combinations of Reynolds number $R e$ and the accept flow rate $\Phi^{+}$: at low $R e$ of 1300 long fibres concentrated in the channel center forming a loose network, which is in line with literature on network formation in straight channel flow. A water annulus was found with small fibres suspended within. For the investigated settings of accept flow rate, only fluid with the suspended fibres was removed from the water annulus. Consequently, a preferred separation of fibres $<1 \mathrm{~mm}$ was achieved. At high $R e$ of 4000 , fibres where dispersed in looser flocs distributed over the whole channel cross section. In this flow regime, freely moving fibres, and/or fibres weakly linked to a floc where removed with the accept flow. Thus, long fibres were removed, but to lower extent than smaller fibres. A minor effect in the tested range of parameters was found for the fibre concentration $C_{\text {feed }}$. For higher $C_{\text {feed }}$ fewer fibres were removed. We speculate that most likely the network strength increased, also hindering smaller fibres from removal with the accept flow.

In summary, the performance of an HDF differs from that of traditional pressure screens, since the former greatly depends on network formation. Similar principles were recently observed in a novel type of hydrocyclone [40]: centrifugal forces add to the formation of a fibre network with this cyclone, which affects fractionation performance. However, no insight into the interplay of Reynolds number $R e$ and accept flow rate $\Phi^{+}$was provided for the 
hydrocyclone in this previous study. In contrast, our present contribution provides first insights into the separation principle of the hydrodynamic fractionation device, namely the interplay of exit layer thickness and network formation near the center of the channel. Future investigations may extend our present work by (i) examining a wider variation of the accept flow rate $\Phi^{+}$for a low Reynolds number $R e$, or (ii) a more detailed investigation on the effect of network formation for different fibres lengths including the relative mobility of larger and shorter fibres.

\section{Acknowledgement}

The authors gratefully acknowledge the industrial partners Sappi Gratkorn, Zellstoff Pöls AG, Norske Skog Bruck, and Mondi Frantschach, the Austrian Research Promotion Agency (FFG), COMET, BMVIT, BMWFJ, the Country of Styria, and Carinthia for their financial support of the K-project FLIPPR.

\section{References}

[1] S. Asikainen, Applicability of fractionation of softwood and hardwood kraft pulp and utilisation of the Applicability of fractionation of softwood and hardwood the fractions, Aalto University, 2015. http://www.vtt.fi/inf/pdf/science/2015/S73.pdf.

[2] M. Karjalainen, Studies on wheat straw pulp fractionation : fractionation tendency of cells in pressure screening, hydrocyclone fractionation and flotation, University of Oulu, 2015. http://urn.fi/urn:isbn:9789526210063.

[3] J.D. Redlinger-Pohn, M. Grabner, P. Zauner, S. Radl, Separation of cellulose fibres from pulp suspension by froth flotation fractionation, Sep. Purif. Technol. 169 (2016) 304-313. doi:10.1016/j.seppur.2016.06.004.

[4] R.W. Gooding, The Passage of Fibres Through Slots in Pulp Screening, The University of British Columbia, 1986. doi:10.14288/1.0058886.

[5] J.A. Olson, The Effect of Fibre Length on Passage Through Narrow Apertures, The University of British Columbia, 1996. doi:10.14288/1.0058501. 
Manuscript - Length-Selective Separation of Cellulose Fibres by HDF

[6] A. Karnis, Pulp fractionation by fibre characteristics, Pap. Ja Puu-Paper Timber. 79 (1997) 480-488.

[7] M. Karjalainen, A. Ammala, P. Rousu, J. Niinimaki, Fractionation of wheat straw pulp in ultra-fine pressure screening, Appita J. 66 (2013) 73-79.

[8] S.J.S. Qazi, M. Mohamad, J.A. Olson, D.M. Martinez, Multistage fiber fractionation of softwood chemical pulp through smooth-hole screen cylinders and its effects on paper properties, TAPPI J. 14 (2015) 259-267.

[9] S. Asikainen, A. Fuhrmann, L. Robertsen, Birch pulp fractions for fine paper and board, Nord. Pulp Pap. Res. J. 25 (2010) 269-276. doi:10.3183/NPPRJ-2010-25-03p269-276.

[10] R.W. Gooding, Flow Resistance of Screen Plate Apertures, The University of British Columbia, 1996. doi:10.14288/1.0058507.

[11] S. Dong, Modelling of Fiber Motion in Pulp and Paper Equipment, The University of British Columbia, 2002. doi:10.14288/1.0103345.

[12] H. Jokinen, Screening and cleaning of pulp: A study to the parameters affecting separation, University of Oulu, 2007. http://urn.fi/urn:isbn:9789514284526.

[13] A. Kumar, Passage of Fibres Through Screen Apertures, The University of British Columbia, 1991. doi:10.14288/1.0059034.

[14] S. Sugaya, M. Yamada, M. Seki, Observation of nonspherical particle behaviors for continuous shape-based separation using hydrodynamic filtration, Biomicrofluidics. 5 (2011) 1-13. doi:10.1063/1.3580757.

[15] C.-T. Huang, P.-N. Li, C.-Y. Pai, T.-S. Leu, C.-P. Jen, Design and Simulation of a Microfluidic Blood-Plasma Separation Chip Using Microchannel Structures, Sep. Sci. Technol. 45 (2009) 42-49. doi:10.1080/01496390903402125.

[16] M. Yamada, M. Seki, Hydrodynamic filtration for on-chip particle concentration and classification utilizing microfluidics., Lab Chip. 5 (2005) 1233-1239. doi:10.1039/b509386d. 
[17] A. Tamura, S. Sugaya, M. Yamada, M. Seki, Tilted-Branch Hydrodynamic Filtration for Length-Dependent Sorting of Rod-Like Particles, in: 15th Int. Conf. Miniaturized Syst. Chem. Life Sci. 2011, 2011: pp. 1343-1345.

[18] M. Walmsley, M. Atkins, Comparing fibre length fractionation of a laboratory flow channel to an industrial pressure screen, in: Proc. 57th Appita Annu. Gen. Conf. Exhib., Melbourne, Australia, 2003: pp. 369-376.

[19] B. Norman, K. Moller, R. Ek, G.G. Duffy, Hydrodynamics of papermaking fibres in water suspensions, in: BPBIF 6th Fund. Res. Symp. Fibre-Water Interact. Papermak., London, 1977: pp. 195-250.

[20] A. Jäsberg, M. Kataja, New Experimental Results on The Flow Regimes in Closed Channel Flows of Wood Fibre Suspensions, in: 14th Fundam. Res. Symp., Oxford, 2009: pp. 161-180.

[21] S. Mokamati, Effect of aperture geometry on the steady flow through the narrow apertures in a pulp screen: numerical and experimental study, The University of British Columbia, 2007. doi:10.14288/1.0080719.

[22] A. Jäsberg, Flow Behaviour of Fibre Suspension in Straight Pipes: New Experimental Techniques and Multiphase Modeling, University of Jyväskylä, 2007. http://urn.fi/URN:ISBN:978-951-39-3187-2.

[23] L.M. König, Particle Separation in a Virtual Microfluidic Channel, Graz University of Technology, 2015.

[24] J. a Olson, Fibre Length Fractionation Caused by Pulp Screening, Slotted Screen Plates, J. Pulp Pap. Sci. 27 (2001) 255-261.

[25] G.B. Jeffery, The Motion of Ellipsoidal Particles Immersed in a Viscous Fluid, Proc. R. Soc. A Math. Phys. Eng. Sci. 102 (1922) 161-179. doi:10.1098/rspa.1922.0078.

[26] J.D. Redlinger-Pohn, L.M. König, C. Kloss, C. Goniva, S. Radl, Modelling of NonSpherical, Elongated Particles for Industrial Suspension Flow Simulation, in: M. Papadrakakis, V. Papadopoulos, G. Stefanou, V. Plevris (Eds.), VII Eur. Congr. Comput. Methods Appl. Sci. Eng., Crete Island, 2016: p. 14. 
Manuscript - Length-Selective Separation of Cellulose Fibres by HDF

[27] C.P.J. Bennington, R.J. Kerekes, J.R. Grace, The yield stress of fibre suspensions, Can. J. Chem. Eng. 68 (1990) 748-757. doi:10.1002/cjce.5450680503.

[28] B. Chen, D. Tatsumi, T. Matsumoto, Fiber Orientation and Flow Properties of Pulp Fiber Suspensions under Shear Flow Conditions, Sen'I Gakkaishi. 59 (2003) 471-478.

[29] B. Derakhshandeh, R.J. Kerekes, S.G. Hatzikiriakos, C.P.J. Bennington, Rheology of pulp fibre suspensions: A critical review, Chem. Eng. Sci. 66 (2011) 3460-3470. doi:10.1016/j.ces.2011.04.017.

[30] M.J. Hourani, Fiber flocculation in pulp suspension flow, Part 1: Theoretical model, TAPPI J. 71 (1988) 115-118.

[31] M.J. Hourani, Fiber flocculation in pulp suspension flow, Part 2: Experimental results, TAPPI J. 71 (1988) 186-189.

[32] H. Cui, J.R. Grace, Flow of pulp fibre suspension and slurries: A review, Int. J. Multiph. Flow. 33 (2007) 921-934. doi:10.1016/j.ijmultiphaseflow.2007.03.004.

[33] R. Meyer, D. Wahren, On the elastic properties of three-dimensional fibre networks, Sven. Papperstidning. 67 (1964) 432-436.

[34] Kerekes, Rheology of fibre suspensions in papermaking: An overview of recent research, Nord. Pulp Pap. Res. J. 21 (2006) 598-612. doi:10.3183/NPPRJ-2006-21-05p598-612.

[35] R.M. Soszynski, R.J. Kerekes, Elastic interlocking of nylon fibers suspended in liquid Part 2. Process of interlocking, Nord. Pulp Pap. Res. J. (1988) 180-184.

[36] D.M. Martinez, H. Kiiskinen, A. Ahlman, R.J. Kerekes, On the Mobility of Flowing Papermaking Suspensions and its Relationship to Formation, 29 (2003) 341-347.

[37] G. Hemström, K. Moller, B. Norman, Boundary layer studies in pulp suspension flow, TAPPI J. 59 (1976) 115-118.

[38] A. Nikbakht, A. Madani, J.A. Olson, D.M. Martinez, Fibre suspensions in HagenPoiseuille flow: Transition from laminar plug flow to turbulence, J. Nonnewton. Fluid Mech. 212 (2014) 28-35. doi:10.1016/j.jnnfm.2014.08.006. 
Manuscript - Length-Selective Separation of Cellulose Fibres by HDF

[39] M. Hortmann, M. Schäfer, Numerical Prediction of Laminiar Flow in Plane Bifurcating Channels, Int. J. Comut. Fluid Dyn. 2 (1994) 65-82. doi:10.1080/10618569408904484.

[40] R. Andersson, H. Vomhoff, Evaluation of a Novel Hydrocyclone Design for Pulp Fractionation, Nord. Pulp Pap. Res. J. 27 (2012) 151-158. doi:10.3183/NPPRJ-201227-01-p151-158.

\section{Appendix}

\subsection{Experimental Settings}

Table 2: Case label and settings. "Geo" states the geometry as: channel height $x$ channel width - angle of the separation channel. The column "Measurement" states the use of a single case (C: concentration, AS: angle of separation channel, Re: Reynolds number, $\Phi$ : flow rate, H: channel height, W: channel width).

\begin{tabular}{|c|c|c|c|c|c|c|c|}
\hline ID & Geo & $\operatorname{Re}$ & $C_{\text {Feed }}$ & $\Phi^{+}$ & Measurement & \multicolumn{2}{|c|}{ Flow Rate } \\
\hline & & & & & & Feed & Accept \\
\hline$[-]$ & {$[-]$} & {$[-]$} & {$[\%]$} & {$[-]$} & {$[-]$} & {$[\mathrm{mL} / \mathrm{min}]$} & {$[\mathrm{mL} / \mathrm{min}]$} \\
\hline 0510-1 & $15 \times 3-20^{\circ}$ & 3476 & 0.487 & 0.019 & $\mathrm{C}$ & 1877 & 35 \\
\hline 0510-2 & $15 \times 3-20^{\circ}$ & 3735 & 0.482 & 0.036 & $\mathrm{C}$ & 2017 & 73 \\
\hline $1610-1$ & $15 \times 3-20^{\circ}$ & 3934 & 0.121 & 0.016 & $\mathrm{G}, \mathrm{Re}, \Phi, \mathrm{H}, \mathrm{W}$ & 2124 & 34 \\
\hline $1610-2$ & $15 \times 3-20^{\circ}$ & 4063 & 0.132 & 0.032 & $\mathrm{C}, \Phi$ & 2194 & 71 \\
\hline $2710-1$ & $15 \times 3-20^{\circ}$ & 1307 & 0.078 & 0.048 & $\mathrm{Re}$ & 706 & 34 \\
\hline 2910-1 & $15 \times 3-90^{\circ}$ & 3800 & .0 .82 & 0.016 & $\mathrm{G}$ & 2052 & 33 \\
\hline $1612-1$ & $9 \times 3-20^{\circ}$ & 1291 & 0.060 & 0.022 & $\mathrm{H}$ & 465 & 10 \\
\hline $1612-2$ & $9 \times 3-20^{\circ}$ & 3745 & 0.129 & 0.015 & $\mathrm{H}$ & 1348 & 20 \\
\hline $2212-1$ & $15 \times 3-20^{\circ}$ & 3881 & 0.113 & 0.017 & $\mathrm{C}, \Phi$ & 2096 & 37 \\
\hline
\end{tabular}


Manuscript - Length-Selective Separation of Cellulose Fibres by HDF

\begin{tabular}{ccccccccc}
\hline $2212-2$ & $15 \times 3-20^{\circ}$ & 1267 & 0.099 & 0.015 & G, Re, H, W & 684 & 10 \\
$1101-1$ & $15 \times 3-90^{\circ}$ & 1283 & 0.098 & 0.015 & G & 693 & 11 \\
$1301-1$ & $15 \times 3-20^{\circ}$ & 3963 & 0.097 & 0.044 & Re, $\Phi$ & 2140 & 94 \\
$1301-2$ & $15 \times 3-20^{\circ}$ & 4131 & 0.112 & 0.005 & $\Phi$ & 2231 & 11 \\
$0303-1$ & $15 \times 9-20^{\circ}$ & 4015 & 0.108 & 0.016 & W & 2891 & 47 \\
$0303-4$ & $15 \times 9-20^{\circ}$ & 1270 & 0.086 & 0.022 & W & 915 & 20 \\
\hline
\end{tabular}

\subsection{Experimental Results}

Table 3: List of results stating the fines separation efficiency $f$, the length-weighted $L_{1}$, and volumeweighted $L_{3}$ mean fibre length. "Red." states the reduction compared to the mean values of the feed.

\begin{tabular}{|c|c|c|c|c|c|}
\hline ID & $f$ & $L_{1, \text { Accept }}$ & $\mathrm{L}_{1}-\mathrm{Red}$ & $L_{3, \text { Accept }}$ & $\mathrm{L}_{3}-$ Red. \\
\hline$[-]$ & {$[\%]$} & {$[\mathrm{mm}]$} & {$[\%]$} & {$[\mathrm{mm}]$} & {$[\%]$} \\
\hline 0510-1 & 0.225 & $0.303 \pm 0.058$ & -81 & $0.830 \pm 0.130$ & -63 \\
\hline 0510-2 & 0.318 & $0.593 \pm 0.053$ & -63 & $1.418 \pm 0.159$ & -38 \\
\hline $1610-1$ & 0.151 & $0.363 \pm 0.070$ & -79 & $0.880 \pm 0.174$ & -63 \\
\hline $1610-2$ & 0.530 & $0.664 \pm 0.005$ & -64 & $1.348 \pm 0.028$ & -44 \\
\hline $2710-1$ & 0.515 & $0.261 \pm 0.081$ & -83 & $0.631 \pm 0.214$ & -72 \\
\hline 2910-1 & 0.130 & $0.266 \pm 0.058$ & -83 & $0.673 \pm 0.165$ & -70 \\
\hline $1612-1$ & 0.081 & $0.129 \pm 0.011$ & -92 & $0.253 \pm 0.076$ & -89 \\
\hline $1612-2$ & 0.068 & $0.185 \pm 0.038$ & -90 & $0.440 \pm 0.140$ & -82 \\
\hline $2212-1$ & 0.174 & $0.408 \pm 0.082$ & -77 & $0.960 \pm 0.121$ & -60 \\
\hline $2212-2$ & 0.023 & $0.109 \pm 0.003$ & -94 & $0.198 \pm 0.009$ & -92 \\
\hline
\end{tabular}


Manuscript - Length-Selective Separation of Cellulose Fibres by HDF

\begin{tabular}{llllll}
\hline $1101-1$ & 0.035 & $0.141 \pm 0.002$ & -92 & $0.300 \pm 0.035$ & -87 \\
$1301-1$ & 1.762 & $1.060 \pm 0.047$ & -40 & $1.834 \pm 0.042$ & -24 \\
$1301-2$ & 0.012 & $0.120 \pm 0.004$ & -93 & $0.200 \pm 0.013$ & -92 \\
$0303-1$ & 0.128 & $0.296 \pm 0.036$ & -83 & $0.714 \pm 0.075$ & -70 \\
$0303-4$ & 0.052 & $0.125 \pm 0.007$ & -93 & $0.274 \pm 0.018$ & -89 \\
\hline
\end{tabular}

